\title{
The trait coding of behavior
}

\author{
MIRIAM J. RODIN \\ San Diego State University, SanDiego, California 92182
}

\begin{abstract}
Trait names are known to convey social desirability information. This study addressed the question of whether they convey denotative behavioral information as well. Encoders assigned trait codings to a variety of possible behavioral responses to well-defined stimulus situations. Decoders then matched the trait codings with the original behaviors. The social desirability of the behaviors was kept relatively constant. It was found that, despite the presence of factors intended to maximize their informativeness, the trait codings transmitted, on the average, only about one-fourth of the input information. The finding that trait codings discriminate poorly among behaviors that do not vary widely in social desirability, in conjunction with the fact that most behavior is socially desirable, raises intriguing questions about the function of trait words in ordinary language.
\end{abstract}

A stranger at a party describes his absent wife as "honest and sincere." The experimenter in an impression formation experiment asks for a likeability judgment of a person who has been described as "kind, generous, and considerate." These instances share two important characteristics: Little or nothing is known about the provider of the information, and the person described by the trait words is unseen and unknown. What kind of information do trait words convey under these conditions?

It has been shown (Rodin, 1972) that trait codings are not very informative when required to differentiate one stimulus person from another. It can be argued, however, that it is completely inappropriate to require of traits that they be informative with respect to the identification of persons. Traits merely code behavioral instances, whereas individuals behave differently at different times with different people. The failure of trait descriptions to be very informative with respect to the identification of stimulus persons may not impugn the trait as a form of coding. It may simply reflect behavioral variability of the stimulus persons toward encoder and decoder.

The present study examined the informativeness of traits with respect to a more realistic criterion: the coding of specific behaviors. The stimulus behaviors were invariant for both encoders and decoders. Encoders assigned trait names to specific instances of behavior. The informativeness of the trait codings was measured by the decoders' ability to recognize which behavioral instance a trait coding was intended to name.

\section{METHOD}

\section{Stimulus Situations}

There were four different stimulus situations. For each stimulus situation, four alternative responses were described. An example of a stimulus situation is: A.B. is playing poker

This paper was sponsored by R. M. Yaremko, who takes full editorial responsibility for its contents. with R. A.B. would like to quit, but $R$. has lost $\$ 10$ and wants a chance to win it back because he is almost broke. A.B. and R. are very good friends. A.B. could react in various ways: (a) He could stop playing and kid $R$. about being a poor loser. (b) He could continue playing and deliberately let $\mathrm{R}$. win back his money. (c) He could stop playing and offer to loan R. the $\$ 10$. (d) He could continue to play for another half hour.

The alternatives pretty well cover the range of possible outcomes; A.B. does or does not continue playing, and R. does or does not get back his money. Missing from the set of alternatives are the socially undesirable versions of the same outcomes; A.B. does not, for example, viciously insult R. about his poor sportsmanship. The selection principle for the alternatives was that they should sample the range of ordinary behavior options without being widely divergent in social desirability, so that this aspect of the alternative could not serve as the recognition marker. It is well known that trait names carry social desirability information (Anderson, 1968). Since the purpose of this study was to determine whether they convey substantive behavioral information as well, it was important to keep the social desirability of the alternatives relatively constant.

\section{Discriminability of the Alternatives}

Did the behavioral alternatives seem essentially alike to the subjects? If so, then of course poor informativeriess of the trait words would be due to the confusability of the stimuli rather than to characteristics of the trait codings. When stimuli are confusable, subjects who are shown one stimulus and later asked to pick it out of an array in which the other stimuli are present should show poor recognition accuracy, particularly when there is an interval of several days between presentation of the initial stimulus and the subsequent recognition task.

Accordingly, a separate group of 16 subjects was asked to provide a trait encoding of one of the behavioral alternatives from each of the four stimulus situations. Each of the four behavioral alternatives in each of the four stimulus situations was encoded by four different subjects. One week later, without forewarning, the subjects were presented with the stimulus situations again and asked to recognize which of the behavior alternatives they had encoded. The behavior alternatives were presented in a different random order on the second administration, so that position cues could not serve as a basis for accurate recognition.

If the behavior alternatives were not discriminable, expected recognition accuracy would be around chance level, or $25 \%$. One of the original 16 subjects was not present at the second administration and was dropped. The average recall accuracy of the remaining 15 subjects was 3.53 out of 4 , or $88 \%$ 
( $p<.01)$. Nine of the 15 subjects had a perfect recall score. It was concluded that the subjects saw the behavior alternatives as discriminably different ways of responding to the stimulus situations.

\section{Encoders}

The 16 encoders were Psychology 1 students recruited from the subject pool. A summary version of the instructions given the encoders is as follows:

On the following pages you will be told about four different situations and some of the ways different people might react in these situations ... As you study a situation, think carefully about the kind of person who would handle the situation in each of these alternative ways. Your job is to find the best word or two to describe each of these different kinds of people ... Try to describe each of these people in such a way that someone else could identify which person you are talking about.

After reading a stimulus situation, the encoder answered the following sort of question: Suppose A.B. were the kind of person who would stop playing and kid R. about being a poor loser. How would you describe this kind of person? (See stimulus situation above.)

The encoders generated their own trait codings. An alternative technique would have been to have encoders choose the trait descriptions from a prior list provided by the experimenter. This procedure was rejected primarily because the informativeness of the codings would clearly depend to some extent on the particular list chosen by the experimenter. In addition, however, having encoders choose from a prescribed list would have made the coding task extremely unnatural: In everyday life codings are freely generated.

All encoders encoded 16 behaviors-the four alternative responses to each of the four stimulus situations. For each stimulus situation, then, there existed 16 encodings for each of the four alternatives. Each encoding was decoded twice. (Eight decoders assigned to a situation each made 16 decoding judgments.)

\section{Decoders}

The 32 decoders were Psychology 1 students recruited from the subject pool. They were randomly and equally divided among the four stimulus situations. The eight decoders assigned to a stimulus situation decoded stimuli only from that one situation.

Each decoder was presented with 16 encodings, four for each of the alternatives. The assignment of encodings was random, with the restriction that no decoder was given identical codings of the same alternative. For example, if two encoders had both chosen the coding "generous" to describe a particular behavioral instance, each of those codings would be assigned to a different decoder. Overlapping codings were those that shared a word or two, e.g., "generous" and "generous and considerate." In all cases but one, it was possible to meet the restriction with the overlapping codings as well. The one exception arose when five overlapping encodings for one of the alternatives had to be divided among four decoders.

The decoders were given the following instructions intended to minimize their tendency to systematically divide the encodings equally among the four response alternatives: ... Each time you make a judgment, it will be collected and you will be given the next one. There is no particular patterning to the answers. Any alternative may be correct any number of times; any alternative may, or may not, be correct several times in succession. The serial judgment procedure described in the instructions was carried out: Encodings were presented one at a time and the prior one collected before the new one was given out.

\section{RESULTS}

The dependent variable was $\hat{T}(x, y)$. This is a measure of the amount of information transmitted from the encoders to the decoders. When no information is transmitted, the (decoder) responses have no relation to the stimuli (encodings) and $\hat{\mathrm{T}}(\mathrm{x}, \mathrm{y})$ equals zero. When information transmission is perfect, each stimulus evokes its own unique response, and $\hat{\mathrm{T}}(\mathrm{x}, \mathrm{y})$ equals the information input. The value of $\hat{T}(x, y)$ may, in other words, vary from a minimum value of zero to a maximum value equal to the amount of information put in. (For further explanation and details of computation, see Attneave, 1957, Chapter 3.)

The information input in each of the situations was 2 bits of information. The amount of information transmitted in the four situations was $.70, .61, .54$, and .29 bits. In the situation for which the codings were most informative, about one-third of the information was transmitted; in the situation for which the codings were least informative, only about one-seventh of the information was transmitted.

\section{DISCUSSION}

It was established in an earlier study that trait names transmitted only about one-fourth of the input information, when the stimuli to be retrieved from the codings were whole stimulus persons. However, since trait names code behavior and the behavior of persons is variable, the ability of trait names to differentiate among stimulus persons may be an inappropriate test of their informativeness. In the present study, the stimuli to be encoded were specific behavioral instances similar in social desirability. Under these conditions, trait names transmitted between one-seventh and one-third of the input information. In short, trait names were inefficient at transmitting even specific behavioral information.

It should be emphasized that every effort was made to maximize transmission efficiency. The encoders had the whole stimulus array in front of them before beginning to code-a procedure that facilitates coding for distinctive features (Boyd, 1969). In addition, the encoders were specifically instructed to code for distinctive features, i.e., to code the behaviors in such a way that someone else would be able to recognize which response alternative was being named. The trait names were freely chosen by the encoders; they did not have to settle for the "best available" on a limited and perhaps biased list provided by the experimenter. The encoding procedure also helped insure that the codings were in a language natural to the student population from which both the encoders and decoders were drawn.

The information required of the trait codings was fairly modest. Decoders were required only to detect which one of several behavior instances was being named. However, trait names presumably do more than name single behaviors; they are ordinarily taken to designate a class of behaviors. To represent $X$ as a "generous" person, in ordinary language, is to make a generalization about his behavior which usually includes future as well as past instances. Trait names would be informative in a fairly deep sense if the person who heard $X$ 's behavior described as "generous" generalized X's behavior over the same class of instances as the speaker. In this study no attempt was made to test whether trait names were informative as generalizations about behavior, only whether they were informative with respect to naming a single instance of behavior.

Would the trait attributions have been more informative if more motivational information were given? In the sample situation, some motivational information was given: The actor wanted to quit, he was a good friend of the other player; the other player wanted to continue the game so that he could recoup his losses. Nevertheless, there is still a great deal of ambiguity. Does A.B. want to quit because he has a headache 
or because he wants the money? If he wants the money, is it for something important or something frivolous? Why is the other person so concerned about losing the money? It is obvious that attribution of motive determines perception of behavior. It is not unlikely that more motivational information would have reduced ambiguity and increased the informativeness of the trait words. With this acknowledgement, however, must go the necessary caveat that motivational information is never complete or unambiguous. The answer to any question seems only to provide information on which to base yet another question. There is another aspect also. Omniscient observers who provide us with motivational information exist only in novels and psychology experiments. In ordinary life motivational information is not simply given-the observer must infer it from behavior, verbal or otherwise. The incompleteness and ambiguity of the motivational information provided to the subjects in this experiment is not a particular feature of the stimuli, but an essential aspect of our motivational knowledge of others. To the question of whether the poor informativeness of the trait codings resulted from a paucity of motivational information, one can only note that the total absence of a scale by which to measure the relative richness of the motivational information effectively precludes an objective answer.

Finally, it might be argued that the trait codings were poorly informative because the $\cdot$ behavioral alternatives sampled only a restricted range of the possible behaviors. Assume all the behavior alternatives in a given stimulus situation could reasonably be called "considerate." Would the experiment then be analogous to one that showed that color names were poorly informative by using closely related shades of blue as stimuli? There are several things to be said in response. First, although it is true that the behavioral alternatives did not vary widely over the spectrum of social desirability, this was an integral aspect of an experiment whose point was to determine whether trait codings convey behavioral as well as social desirability information. If the behavioral alternatives varied widely in social desirability, then obtained discrimination among judges could as readily have been ascribed to the social desirability information as to the denotative information conveyed by the trait names. Second, the fact that two behaviors could both be called "considerate" is beside the point when the question is whether there exist trait names that can reliably communicate to others which behavior is which, just as the fact that two objects in this room can both be called furniture is irrelevant to the question of whether there exist nouns that can reliably differentiate the chair from the table. The last, and probably most important, point is that most people behave in a socially desirable manner most of the time. ${ }^{1}$ Ordinary behavior occupies only a limited range of possible behavior. The informativeness of trait names over a limited range of social desirability thus speaks to their informativeness in usual circumstances.

Trait names conveyed little substantive behavioral information, even though encoding distinctiveness was maximized by allowing judges to see the whole array before beginning to encode, judges were allowed to generate their own encodings rather than forced to select from an arbitrary list, motivational information was provided, and the informativeness criterion was minimal. That trait names do not differentiate well among behaviors that are similar in social desirability implies that trait names do not discriminate among ordinary behaviors. What then might be their function in that restricted set of circumstances in which the person receiving the information knows little or nothing about the person providing the trait description and the person being described is unseen and unknown. It is tempting to speculate that the ordinary inference from this sort of information is to the person describing rather than to the person described. That is, perhaps the natural inference from trait information in these circumstances is to the speaker's evaluative impression of the targei person rather than to any substantive impression of the target person per se.

\section{REFERENCES}

ANDERSON. N. H. Likeableness ratings of 555 personality trait words. Journal of Personality and Social Psychology, 1968, 9. 272-279.

Attneave, F. Applications of information theory to psychology. New York: Holt-Dryden, 1957.

BoYD. J. E. Efficiency of communicating personality impressions as a function of coding context. Psychonomic Science, 1969, 14. 57-58.

Rodin, M. J. The informativeness of trait descriptions. Journal of Personality and Social Psychology, 1972, 21, $341-344$.

\section{NOTE}

1. I am most indebted to David Schneider for bringing the relevance of this fact to my attention.

(Received for publication August 29, 1975.) 